\title{
Octonionic Ternary Gauge Field Theories Revisited
}

\author{
Carlos Castro \\ Center for Theoretical Studies of Physical Systems \\ Clark Atlanta University, Atlanta, Georgia. 30314, perelmanc@hotmail.com
}

May 2013; Revised July 2013

\begin{abstract}
An octonionic ternary gauge field theory is explicitly constructed based on a ternary-bracket defined earlier by Yamazaki. The ternary infinitesimal gauge transformations do obey the key closure relations $\left[\delta_{1}, \delta_{2}\right]=\delta_{3}$. An invariant action for the octonionic-valued gauge fields is displayed after solving the previous problems in formulating a non-associative octonionic ternary gauge field theory. These octonionc ternary gauge field theories constructed here deserve further investigation. In particular, to study their relation to Yang-Mills theories based on the $G_{2}$ group which is the automorphism group of the Octonions and their relevance to Noncommutative and Nonassociative Geometry.
\end{abstract}

\section{Introduction}

Exceptional, Jordan, Division, Clifford, noncommutative and nonassociative algebras are deeply related and are essential tools in many aspects in Physics, see [1], [2], [3], [4], [5], [6], [7], [8], [8], [12], [13], [14], [25], [26] for references, among many others. A thorough discussion of the relevance of ternary and nonassociative structures in Physics has been provided in [10], [15], [16],[17],[18], [19]. The earliest example of nonassociative structures in Physics can be found in Einstein's special theory of relativity. Only colinear velocities are commutative and associative, but in general, the addition of non-colinear velocities is non-associative and non-commutative.

Great activity was launched by the seminal works of Bagger, Lambert and Gustavsson (BLG) [27], [28], [29] who proposed a Chern-Simons type Lagrangian describing the world-volume theory of multiple $M 2$-branes. The original BLG theory requires the algebraic structures of generalized Lie 3-algebras and also of nonassociative algebras. Later developments by [30] provided a $3 D$ ChernSimons matter theory with $\mathcal{N}=6$ supersymmetry and with gauge groups 
$U(N) \times U(N), S U(N) \times S U(N)$. The original construction of [30] did not require generalized Lie 3 -algebras, but it was later realized that it could be understood as a special class of models based on Hermitian 3-algebras [31], [32].

A Nonassociative Gauge theory based on the Moufang $S^{7}$ loop product (not a Lie algebra) has been constructed by [33], [34]. Taking the algebra of octonions with a unit norm as the Moufang $S^{7}$-loop, one reproduces a nonassociative octonionic gauge theory which is a generalization of the Maxwell and YangMills gauge theories based on Lie algebras. BPST-like instantons solutions in $D=8$ were also found. These solutions represented the physical degrees of freedom of the transverse 8-dimensions of superstring solitons in $D=10$ preserving one and two of the 16 spacetime supersymmetries. Nonassociative deformations of Yang-Mills Gauge theories involving the left and right bimodules of the octonionic algebra were presented by [35]. Non-associative generalizations of supersymmetry have been proposed by [36] which is very relevant to hidden variables theory and alternative Quantum Mechanics.

The ternary gauge theory developed in this work differs from the work by [27], [28], [29], [33], [34] in that our 3-Lie algebra-valued gauge field strengths $F_{\mu \nu}$ are explicitly defined in terms of a 3-bracket $\left[A_{\mu}, A_{\nu}, \mathbf{g}\right]$ involving a 3-Lie algebravalued coupling $\mathbf{g}=g^{a} t_{a}$. Whereas the definition of $F_{\mu \nu}$ by [27], [28], [29] was based on the standard commutator of the matrices $\left(\tilde{A}_{\mu}\right)_{c}^{a}\left(\tilde{A}_{\nu}\right)_{b}^{c}-\left(\tilde{A}_{\nu}\right)_{c}^{a}\left(\tilde{A}_{\mu}\right)_{b}^{c}$. These matrices were defined as $\mathbf{A}_{\mu}=A_{\mu}^{a b} f_{a b}{ }^{c d}=\left(\tilde{A}_{\mu}\right)^{c d}$ and given in terms of the structure constants $f_{a b}{ }^{c d}$ of the 3-Lie algebra $\left[t_{a}, t_{b}, t^{c}\right]=f_{a b}{ }^{c d} t_{d}$.

In the next section we shall analyze Nonassociative Octonionic Ternary Gauge Field Theories based on a ternary octonionic product with the fundamental difference, besides the nonassociativity, that the structure constants $f_{a b c d}$ are no longer totally antisymmetric in their indices. Thus the bracket in the octonion case $[[A, B]] \equiv[A, B, \mathbf{g}]$ is not effectively a Lie bracket (as it occurs in the 3 -Lie algebra case) because the bracket $[[A, B]]$ in the octonion case does not obey the Jacobi identity since the structure constants $f_{a b c d}$ are no longer totally antisymmetric in their indices. This work is quite an improvement of our prior results where we focused solely on the global rigid symmetries and homothecy transformations [38].

It is shown that the octonionic-valued field strength $F_{\mu \nu}=F_{\mu \nu}^{a} e_{a}$ transforms homogeneously (covariantly) under gauge transformations and that the YangMills-like action is indeed invariant under local gauge transformations involving ternary octonionic brackets and antisymmetric gauge parameters $\Lambda^{a b}(x)=$ $-\Lambda^{b a}(x), a, b=0,1,2,3, \ldots .7$. Furthermore, there is closure of these transformations based on antisymmetric parameters $\Lambda^{a b}=-\Lambda^{b a}$

\section{Octonionic Ternary Gauge Field Theories}

The nonassociative and noncommutative octonionic ternary gauge field theory is based on a ternary-bracket structure involving the octonion algebra. The 
ternary bracket obeys the fundamental identity (generalized Jacobi identity) and was developed earlier by Yamazaki [24]. Given an octonion $\mathbf{X}$ it can be expanded in a basis $\left(e_{o}, e_{m}\right)$ as

$$
\mathbf{X}=x^{o} e_{o}+x^{m} e_{m}, \quad m, n, p=1,2,3, \ldots . .7 .
$$

where $e_{o}$ is the identity element. The Noncommutative and Nonassociative algebra of octonions is determined from the relations

$$
e_{o}^{2}=e_{o}, e_{o} e_{i}=e_{i} e_{o}=e_{i}, e_{i} e_{j}=-\delta_{i j} e_{o}+c_{i j k} e_{k}, i, j, k=1,2,3, \ldots .7 .
$$

where the fully antisymmetric structure constants $c_{i j k}$ are taken to be 1 for the combinations (124), (235), (346), (457), (561), (672), (713). The octonion conjugate is defined by $\bar{e}_{o}=e_{o}, \bar{e}_{i}=-e_{i}$

$$
\overline{\mathbf{X}}=x^{o} e_{o}-x^{k} e_{k} .
$$

and the norm is

$$
N(\mathbf{X})=|<\mathbf{X ~ X}>|^{\frac{1}{2}}=|\operatorname{Real}(\overline{\mathbf{X}} \mathbf{X})|^{\frac{1}{2}}=\left|\left(x_{o} x_{o}+x_{k} x_{k}\right)\right|^{\frac{1}{2}} .
$$

The inverse

$$
\mathbf{X}^{-1}=\frac{\overline{\mathbf{X}}}{<\mathbf{X} \mathbf{X}>}, \quad \mathbf{X}^{-1} \mathbf{X}=\mathbf{X X}^{-1}=1 .
$$

The non-vanishing associator is defined by

$$
(\mathbf{X}, \mathbf{Y}, \mathbf{Z})=(\mathbf{X Y}) \mathbf{Z}-\mathbf{X}(\mathbf{Y Z})
$$

In particular, the associator

$$
\begin{gathered}
\left(e_{i}, e_{j}, e_{k}\right)=\left(e_{i} e_{j}\right) e_{k}-e_{i}\left(e_{j} e_{k}\right)=2 d_{i j k l} e_{l} \\
d_{i j k l}=\frac{1}{3 !} \epsilon_{i j k l m n p} c^{m n p}, i, j, k \ldots=1,2,3, \ldots . .7
\end{gathered}
$$

Yamazaki [24] defined the three-bracket as

$[u, v, x] \equiv D_{u, v} x=\frac{1}{2}(u(v x)-v(u x)+(x v) u-(x u) v+u(x v)-(u x) v)$.

After a straightforward calculation when the indices span the imaginary elements $a, b, c, d=1,2,3, \ldots \ldots ., 7$, and using the relationship [37]

$$
c_{a b d} c_{d c m}=-d_{a b c m}+\delta_{a c} \delta_{b m}-\delta_{b c} \delta_{a m}
$$

the ternary bracket becomes

$$
\left[e_{a}, e_{b}, e_{c}\right]=f_{a b c d} e_{d}=\left[d_{a b c d}+2 \delta_{a c} \delta_{b d}-2 \delta_{b c} \delta_{a d}\right] e_{d}
$$


whereas $e_{0}$ has a vanishing ternary bracket

$$
\left[e_{a}, e_{b}, e_{0}\right]=\left[e_{a}, e_{0}, e_{b}\right]=\left[e_{0}, e_{a}, e_{b}\right]=0
$$

It is important to emphasize that $f_{a b c d} \neq \pm c_{a b d} c_{d c m}$ otherwise one would have been able to rewrite the ternary bracket in terms of ordinary 2-brackets as follows $\left[e_{a}, e_{b}, e_{c}\right] \sim \frac{1}{4}\left[\left[e_{a}, e_{b}\right], e_{c}\right]$ and this would have defeated the whole purpose of studying ternary structures.

The ternary bracket (2.8) obeys the fundamental identity

$[[x, u, v], y, z]+[x,[y, u, v], z]+[x, y,[z, u, v]]=[[x, y, z], u, v]$

A bilinear positive symmetric product $\langle u, v\rangle=\langle v, u\rangle$ is required such that that the ternary bracket/derivation obeys what is called the metric compatibility condition

$$
\begin{gathered}
<[u, v, x], y>=-<[u, v, y], x>=-<x,[u, v, y]>\Rightarrow \\
D_{u, v}<x, y>=0
\end{gathered}
$$

The symmetric product remains invariant under derivations. There is also the additional symmetry condition required by [24]

$$
<[u, v, x], y>=<[x, y, u], v>
$$

The ternary product provided by Yamazaki (2.8) obeys the key fundamental identity (2.10) and leads to the structure constants $f_{a b c d}$ that are pairwise antisymmetric but are not totally antisymmetric in all of their indices : $f_{a b c d}=$ $-f_{b a c d}=-f_{a b d c}=f_{c d a b}$; however : $f_{a b c d} \neq f_{c a b d}$; and $f_{a b c d} \neq-f_{d b c a}$. The associator ternary operation for octonions $(x, y, z)=(x y) z-x(y z)$ does not obey the fundamental identity (2.10) as emphasized by [24]. For this reason we cannot use the associator to construct the 3-bracket.

The physical motivation behind constructing an octonionic-valued field strength in terms of ternary brackets is because the ordinary 2-bracket does not obey the Jacobi identity

$$
\left[e_{i},\left[e_{j}, e_{k}\right]\right]+\left[e_{j},\left[e_{k}, e_{i}\right]\right]+\left[e_{k},\left[e_{i}, e_{j}\right]\right]=3 d_{i j k l} e_{l} \neq 0
$$

If one has the ordinary Yang-Mills expression for the field strength

$$
F_{\mu \nu}=\partial_{\mu} A_{\nu}-\partial_{\nu} A_{\mu}+\left[A_{\mu}, A_{\nu}\right]
$$

because the 2-bracket does not obey the Jacobi identity, one has an extra (spurious) term in the expression for

$$
\left[D_{\mu}, D_{\nu}\right] \Phi=\left[F_{\mu \nu}, \Phi\right]+\left(A_{\mu}, A_{\nu}, \Phi\right)
$$


given by the crucial contribution of the non-vanishing associator $\left(A_{\mu}, A_{\nu}, \Phi\right)=$ $\left(A_{\mu} A_{\nu}\right) \Phi-A_{\mu}\left(A_{\nu} \Phi\right) \neq 0$. For this reason, due to the non-vanishing condition (2.13), the ordinary Yang-Mills field strength does not transform homogeneously under ordinary gauge transformations involving the parameters $\Lambda=\Lambda^{a} e_{a}$

$$
\delta A_{\mu}=\partial_{\mu} \Lambda+\left[A_{\mu}, \Lambda\right]
$$

but it yields an extra contribution of the form

$$
\delta F_{\mu \nu}=\left[F_{\mu \nu}, \Lambda\right]+\left(\Lambda, A_{\mu}, A_{\nu}\right)
$$

As a result of the additional contribution $\left(\Lambda, A_{\mu}, A_{\nu}\right)$ in eq- $(2.17)$, the ordinary Yang-Mills action $S=\int\left\langle F_{\mu \nu} F^{\mu \nu}>\right.$ will no longer be gauge invariant. Under infinitesimal variations (2.17), the variation of the action is no longer zero but receives spurious contributions of the form $\delta S=-4 F_{\mu \nu}^{l} \Lambda^{i} A^{\mu j} A^{\nu k} d_{i j k l} \neq 0$ due to the non-associativity of the octonion algebra. For these reasons we focus our attention on ternary brackets.

We define the field strength in terms of the ternary bracket as

$$
F_{\mu \nu}=\partial_{\mu} A_{\nu}-\partial_{\nu} A_{\mu}+\left[A_{\mu}, A_{\nu}, \mathbf{g}\right]
$$

where $\mathbf{g}=g^{a} e_{a}$ is an octonionic-valued "coupling" function. One finds that under the naive infinitesimal ternary gauge transformations

$\delta\left(A_{\mu}^{d} e_{d}\right)=-\partial_{\mu}\left(\Lambda^{d} e_{d}\right)+\left[\Lambda^{a} e_{a}, A_{\mu}^{b} e_{b}, g^{c} e_{c}\right] \Rightarrow \delta\left(F_{\mu \nu}^{d} e_{d}\right)=\left[\Lambda^{a}(x) e_{a}, F_{\mu \nu}^{b} e_{b}, g^{c} e_{c}\right]$

the ordinary quadratic action

$$
S=-\frac{1}{4 \kappa^{2}} \int d^{D} x<F_{\mu \nu} F^{\mu \nu}>
$$

is not invariant under ternary infinitesimal gauge transformations as we shall see next. $\kappa$ is a suitable dimensionful constant introduced to render the action dimensionless. The octonionic valued field strength is $F_{\mu \nu}=F_{\mu \nu}^{a} e_{a}$, and has real valued components $F_{\mu \nu}^{0}, F_{\mu \nu}^{i} ; i=1,2,3, \ldots . ., 7$. The $<>$ operation extracting the $e_{0}$ part is defined as $\langle X Y\rangle=\operatorname{Real}(\bar{X} Y)=<Y X>=\operatorname{Real}(\bar{Y} X)$. Under infinitesimal ternary gauge transformations of the ordinary quadratic action one has

$$
\begin{gathered}
\delta S=-\frac{1}{4 \kappa^{2}} \int d^{D} x<F_{\mu \nu}\left(\delta F^{\mu \nu}\right)+\left(\delta F_{\mu \nu}\right) F^{\mu \nu}>= \\
-\frac{1}{4 \kappa^{2}} \int d^{D} x<F_{\mu \nu}^{c} e_{c}\left[\Lambda^{a} e_{a}, F^{\mu \nu b} e_{b}, g^{n} e_{n}\right]+ \\
-\frac{1}{4 \kappa^{2}} \int d^{D} x<\left[\Lambda^{a} e_{a}, F_{\mu \nu}^{b} e_{b}, g^{n} e_{n}\right] F^{\mu \nu c} e_{c}>= \\
-\frac{1}{4 \kappa^{2}} \int d^{D} x \Lambda^{a} F_{\mu \nu}^{c} F^{\mu \nu b}\left(<e_{c} f_{a b n k} e_{k}>+<f_{a b n k} e_{k} e_{c}>\right)=
\end{gathered}
$$




$$
\begin{gathered}
-\frac{1}{2 \kappa^{2}} \int d^{D} x \Lambda^{a} g^{n} F_{\mu \nu}^{c} F^{\mu \nu b} f_{a b n c}= \\
-\frac{1}{\kappa^{2}} \int d^{D} x\left(\left(\Lambda^{a} g_{a}\right)\left(F_{\mu \nu}^{b} F_{b}^{\mu \nu}\right)-\left(\Lambda^{a} F_{a}^{\mu \nu}\right)\left(g_{c} F_{\mu \nu}^{c}\right)\right) \neq 0
\end{gathered}
$$

Hence, because

$$
f_{a b n c}=\left(d_{a b n c}+2 \delta_{a n} \delta_{b c}-2 \delta_{b n} \delta_{a c}\right)
$$

is not antisymmetric under the exchange of indices $b \leftrightarrow c: f_{a b n c} \neq-f_{a c n b}$, the variation in eq-(3.15) is not zero. Had $f_{a b n c}$ been fully antisymmetric then the variation $\delta S$ would have been zero due to the fact that $F_{\mu \nu}^{c} F^{\mu \nu b}$ is symmetric under $b \leftrightarrow c$.

Concluding, in the octonionic ternary algebra case, the naive transformations (2.19) do not leave the action (2.20) invariant : $\delta S \neq 0$. One alternative would be to find counter terms, if possible, to the action (2.20) $S+\Delta S$ so that $\delta(S+$ $\Delta S)=0$. The authors [35] used counter terms of the form $F \wedge A \wedge A+A \wedge A \wedge A \wedge A$ in the non-associative deformations of ordinary Yang-Mills theories based on the left and right actions by octonions and ordinary brackets. Unfortunately it does not work in our case and for this reason we shall follow a different approach.

Another problem due to the fact that $f_{a b c d}$ is not totally antisymmetric in all of its indices is that there is no closure of the infinitesimal octonionic ternary gauge transformations $\delta A_{\mu}=-\partial_{\mu} \Lambda+\left[\Lambda, A_{\mu}, \mathbf{g}\right]$. Furthermore, the bracket in the octonion case $[[A, B]] \equiv[A, B, \mathbf{g}]$ is not effectively a Lie bracket (as it is in the 3 -Lie algebra case) since the bracket $[[A, B]]$ in the octonion case does not obey the Jacobi identity because the structure constants $f_{a b c d}$ are no longer totally antisymmetric in their indices. As said previously, because the associator ternary operation for octonions $(x, y, z)=(x y) z-x(y z)$ does not obey the fundamental identity (3.10) one cannot use the associator to construct the 3-bracket and this rules out the use of the totally antisymmetric $d_{a b c d}$.

Nevertheless, as we shall show below, the quadratic Yang-Mills-like action (2.20) is invariant under the local octonionic ternary gauge transformations defined by

and

$$
\delta\left(A_{\mu}^{d} e_{d}\right)=\Lambda^{a b}(x)\left[e_{a}, e_{b}, A_{\mu}^{c} e_{c}\right]
$$

$$
\delta\left(g^{d} e_{d}\right)=\Lambda^{a b}(x)\left[e_{a}, e_{b}, g^{c} e_{c}\right]
$$

where one introduces a local spacetime depedence on the antisymmetric gauge parameters $\Lambda^{a b}(x)=-\Lambda^{b a}(x)$. One may notice now that the coupling $g^{c} e_{c}$ is not inert under the transformations (2.23b). Only the real part of the coupling $g^{0}$ is inert.

After some straightforward algebra one can verify that the ternary field strength $F_{\mu \nu}$ defined in terms of the 3-brackets transforms properly (homogeneously) under the local transformations (2.23)

$\delta\left(F_{\mu \nu}^{m} e_{m}\right)=\Lambda^{a b}\left[e_{a}, e_{b}, F_{\mu \nu}^{c} e_{c}\right]=\Lambda^{a b} F_{\mu \nu}^{c} f_{a b c}{ }^{m} e_{m} \Rightarrow \delta F_{\mu \nu}^{m}=\Lambda^{a b} F_{\mu \nu}^{c} f_{a b c}{ }^{m}$ 
if the following conditions are satisfied

$$
\left[\left(\partial_{\mu} \Lambda^{i j}\right) A_{\nu}^{k}-\left(\partial_{\nu} \Lambda^{i j}\right) A_{\mu}^{k}\right] f_{i j k}^{l}=0
$$

Due to the key presence of the imaginary parts of the couplings $g^{i}$ we shall prove below that one can partially gauge the fields by setting $A_{\mu}^{i}=g^{i}(x) \partial_{\mu} \phi(x)$ (in terms of an auxiliary scalar field $\phi(x)$ ) and still leave room for residual symmetries. One should note that even if the coupling functions $g^{i}$ are chosen to be constants $g^{i}=$ constant, it must be kept in mind that after gauge transformations the new couplings $g^{i}$ will acquire a spacetime dependence via the $x^{\mu}$-dependence of the $\Lambda^{a b}\left(x^{\mu}\right)$ parameters. For this reason we should not set a priori the couplings to constants. Furthermore, the field strength will not become trivially zero. It can be rewritten, when the gauge fields are partially gauged as $A_{\mu}^{l}=g^{l}(x) \partial_{\mu} \phi(x)$, in the following way

$$
F_{\mu \nu}^{l}=\partial_{[\mu} A_{\nu]}^{l}+A_{\mu}^{i} A_{\nu}^{j} g^{k} f_{i j k}^{l}=\left(\partial_{\mu} g^{l}\right)\left(\partial_{\nu} \phi\right)-\left(\partial_{\nu} g^{l}\right)\left(\partial_{\mu} \phi\right) \neq 0
$$

after using the conditions $\left(g^{i} \partial_{\mu} \phi\right)\left(g^{j} \partial_{\nu} \phi\right) f_{i j k}^{l}=0$ due to the antisymmetry of $f_{i j k}^{l}=-f_{j i k}^{l}$ and the symmetry $g^{i} g^{j}=g^{j} g^{i}$. Therefore, due to the $x$ dependence of the imaginary parts of the couplings $g^{i}(x)$, the field strength components $F_{\mu \nu}^{l}$ are not zero and their contribution to the action (2.20) is not trivially zero.

After this detour let us look for nontrivial solutions to (2.25). $\Lambda^{i j}=$ constant are the trivial solutions leading to global rigid symmetries. A partial gauge fixing provided by $A_{\mu}^{k}=g^{k} \partial_{\mu} \phi, A_{\nu}^{k}=g^{k} \partial_{\nu} \phi$ yields in eq-(2.25), after factoring $g^{k}$ and defining $\tilde{\Lambda}_{k}^{l}=f_{i j k}{ }^{l} \Lambda^{i j}$, the following equation

$$
\left(\partial_{\mu} \tilde{\Lambda}_{k}^{l}\right)\left(\partial_{\nu} \phi\right)-\left(\partial_{\nu} \tilde{\Lambda}_{k}^{l}\right)\left(\partial_{\mu} \phi\right)=0
$$

eq-(2.27) can also be rewritten as

$$
\partial_{\mu}\left(\tilde{\Lambda}_{k}^{l} \partial_{\nu} \phi\right)-\partial_{\nu}\left(\tilde{\Lambda}_{k}^{l} \partial_{\mu} \phi\right)=0
$$

a solution to eq- $(2.27)$ can be easily obtained when

$$
\partial_{\mu} \tilde{\Lambda}_{k}^{l}=\lambda \tilde{\Lambda}_{k}^{l} \partial_{\mu} \phi, \partial_{\nu} \tilde{\Lambda}_{k}^{l}=\lambda \tilde{\Lambda}_{k}^{l} \partial_{\nu} \phi \Rightarrow \tilde{\Lambda}_{k}^{l}(x)=\tilde{C}_{k}^{l} e^{\lambda \phi(x)}, \lambda \neq 0(2.29 a)
$$

$\lambda$ is an arbitrary nonzero constant. Since $\tilde{\Lambda}_{k}^{l}=f_{i j k}{ }^{l} \Lambda^{i j}$ one can infer that

$$
\Lambda^{i j}(x)=C^{i j} e^{\lambda \phi(x)}, \quad \tilde{C}_{k}^{l}=f_{i j k}^{l} C^{i j}, \quad \lambda \neq 0
$$

where $\tilde{C}_{k}^{l}$ and $C^{i j}$ are the constant entries of the arbitrary constant $7 \times 7$ matrices $\tilde{\mathbf{C}}, \mathbf{C}$ when the range of indices is $l, k=1,2,3, \ldots \ldots, 7$. Inserting the solutions (2.29a) back into eq-(2.27) one immediately can verify that

$$
\lambda \tilde{\Lambda}_{k}^{l}\left(\partial_{\mu} \phi \partial_{\nu} \phi-\partial_{\nu} \phi \partial_{\mu} \phi\right)=0
$$


therefore, the expression $\tilde{\Lambda}_{k}^{l}(x)=\tilde{C}_{k}^{l} e^{\lambda \phi(x)}$ is indeed a correct and nontrivial solution to eq-(2.27). When $\lambda=0$ one recovers in eqs-(2.29a, 2.29b) the trivial solutions $\tilde{\Lambda}_{k}^{l}=$ constants, $\Lambda^{i j}=$ constants corresponding to the global (rigid) transformations.

To sum up, after partially gauging the field

$$
A_{\mu}^{k}(x)=g^{k}(x) \partial_{\mu} \phi
$$

the gauge parameters which solve eq-(2.25) are given by $\Lambda^{i j}(x)=C^{i j} e^{\lambda \phi(x)}$ and, consequently, one has found nontrivial solutions for the gauge parameters. From eq- $(2.30)$ one can infer the explicit relation between $\phi$ and $A_{\mu}^{k}$ given by the following line integral

$$
\phi-\phi_{o}=\int_{0}^{x^{\mu}} \frac{g_{k}\left(x^{\prime \rho}\right) A_{\mu}^{k}\left(x^{\prime \rho}\right)}{g^{2}\left(x^{\prime \rho}\right)} d x^{\prime \mu}
$$

The exponential of the constant $\phi_{o} \equiv \phi\left(x^{\mu}=0\right)$ can be reabsorbed into the definition of the entries of the constant matrix so that the field dependence of the parameters becomes

$$
\Lambda^{i j}\left(x^{\mu}\right)=C^{i j} e^{\lambda \phi\left(x^{\mu}\right)}=C^{i j} \exp \left(\lambda \int_{0}^{x^{\mu}} \frac{g_{k}\left(x^{\prime \rho}\right) A_{\mu}^{k}\left(x^{\prime \rho}\right)}{g^{2}\left(x^{\prime \rho}\right)} d x^{\prime \mu}\right)
$$

and the gauge transformations (2.23) are now nonlinear in the fields. Hence, we finally have arrived at eqs- $(2.30,2.31 \mathrm{~b})$ which are the nontrivial solutions to eq- $(2.25)$ when $\lambda \neq 0$. It is interesting that (2.31b) is given by an expression which resembles the Wilson loop form with the main difference that one has a line integral instead of a loop. The value of the line integral is path-independent and solely depends on the initial point (the origin of coordinates) and the final point $x^{\mu}$.

To conclude, when one partially gauges the fields as $A_{\mu}^{k}=g^{k} \partial_{\mu} \phi$, there is still a residual symmetry that remains such that the gauge transformations (2.23) become nonlinear in the fields and the nonvanishing field strength $F_{\mu \nu}^{k}$ given by eq-(2.26) transforms homogeneously (2.24) under the local gauge transformations (2.23) due to the vanishing conditions imposed by eq-(2.25). The component associated with the unit element of the octonion algebra $F_{\mu \nu}^{0}$ is inert $\delta F_{\mu \nu}^{0}=0$ under the gauge transformations.

The reason the field strength $F_{\mu \nu}^{k}$ transforms homogeneously (2.24) when the inhomogeneous terms (2.25) vanish is a direct consequence of the fundamental identity (2.10) because the 3 -bracket (2.8) is defined as a derivation

$$
\begin{gathered}
{\left[\left[e_{a}, e_{b}, A_{\mu}\right], A_{\nu}, \mathbf{g}\right]+\left[A_{\mu},\left[e_{a}, e_{b}, A_{\nu}\right], \mathbf{g}\right]+\left[A_{\mu}, A_{\nu},\left[e_{a}, e_{b}, \mathbf{g}\right]\right]=} \\
{\left[e_{a}, e_{b},\left[A_{\mu}, A_{\nu}, \mathbf{g}\right]\right]}
\end{gathered}
$$


Another important condition due to the antisymmetry $f_{i j k l}=-f_{j i k l}=-f_{i j l k}$, and symmetry $g^{l} g^{k}=g^{k} g^{l}$, is the invariance of $g^{2}=g_{l} g^{l}$ under gauge transformations

$$
\delta g^{2}=\delta\left(g_{l} g^{l}\right)=2 g_{l} \delta g^{l}=2 g_{l} \Lambda^{i j} g^{k} f_{i j k}^{l}=2 g^{l} g^{k} \Lambda^{i j} f_{i j k l}=0
$$

Therefore, the full octonionic norm-squared $\left(g^{o}\right)^{2}+g_{i} g^{i}$ of the octonionic-valued coupling function $\mathbf{g}=g^{o} e_{o}+g^{i} e_{i}$ is invariant under gauge transformations (2.23b).

An important remark is in order. There is a plausible caveat about the conditions (2.25). One must ensure that such conditions, which do not appear to be explicitly gauge covariant, will not break the gauge covariance (invariance) of the theory one is trying to construct. In particular, after performing a gauge variation of the conditions $\mathcal{C}_{[\mu \nu]}^{l}=0$ in $(2.25)$ one would introduce the secondary conditions $\delta \mathcal{C}_{[\mu \nu]}^{l}=0$. Performing yet another gauge variation of the secondary conditions $\delta\left(\delta \mathcal{C}_{[\mu \nu]}^{l}\right)=0 \ldots$, and so forth, one obtains a hierarchy of equations to be satisfied by the gauge parameters $\Lambda^{i j}(x)$. It is clear that the trivial solutions $\Lambda^{i j}=C^{i j}=$ constants will satisfy automatically all the equations suggesting, perhaps, that octonionic ternary field theories cannot be gauged. Nevertheless, as we shall show next, there is a very natural way to bypass this problem such that the gauge variation of the conditions (2.25) remains zero without introducing additional constraints on the parameters $\Lambda^{i j}$ that might have forced them to be constants. From the gauge variations

$$
\begin{gathered}
\delta A_{\mu}^{l}=\Lambda^{i j}(x) A_{\mu}^{k} f_{i j k}^{l}=\Lambda^{i j}(x) g^{k} \partial_{\mu} \phi f_{i j k}^{l}=\delta\left(g^{l} \partial_{\mu} \phi\right)= \\
\delta\left(g^{l}\right) \partial_{\mu} \phi+g^{l} \partial_{\mu}(\delta \phi)=\Lambda^{i j}(x) g^{k} \partial_{\mu} \phi f_{i j k}^{l}+g^{l} \partial_{\mu}(\delta \phi) \Rightarrow \delta \phi=0
\end{gathered}
$$

one learns that $\delta \phi=0$, and in turn, we can infer from (2.31b) that $\delta \Lambda^{i j}=0$ so that the variation of the condition (2.25) (when $A_{\mu}^{i}=g^{i} \partial_{\mu} \phi, \delta \Lambda^{i j}=\delta \phi=0$ ) remains zero without introducing further constraints on the parameters. A variation of (2.25) gives then

$$
f_{i j k}^{l}\left(\left(\partial_{\mu} \Lambda^{i j}\right)\left(\delta g^{k}\right)\left(\partial_{\nu} \phi\right)-\left(\partial_{\nu} \Lambda^{i j}\right)\left(\delta g^{k}\right)\left(\partial_{\mu} \phi\right)\right)=0
$$

therefore, after simple factorization of $\delta g^{k}$ in (2.35) it leads to the exact same equation (2.27) at the beginning obtained from a factorization of $g^{k}$ in (2.25) and which admits the solutions described above. Hence, the variations of (2.25) do not impose additional constraints on the parameters $\Lambda^{i j}$ which might have forced us to have the trivial constant solutions for these gauge parameters.

Finally, given the octonionic valued field strength $F_{\mu \nu}=F_{\mu \nu}^{a} e_{a}$, with real valued components $F_{\mu \nu}^{0}, F_{\mu \nu}^{i} ; i=1,2,3, \ldots . ., 7$, one can verify that the quadratic action (2.20) is indeed invariant under the ternary infinitesimal local gauge transformations (2.23) when the field strength transforms as provided by eq- $(2.24)$

$$
\delta S=-\frac{1}{4 \kappa^{2}} \int d^{D} x<F_{\mu \nu}\left(\delta F^{\mu \nu}\right)+\left(\delta F_{\mu \nu}\right) F^{\mu \nu}>=
$$




$$
\begin{gathered}
-\frac{1}{4 \kappa^{2}} \int d^{D} x<F_{\mu \nu}^{c} e_{c} \Lambda^{a b}\left[e_{a}, e_{b}, F^{\mu \nu n} e_{n}\right]>+ \\
-\frac{1}{4 \kappa^{2}} \int d^{D} x<\Lambda^{a b}\left[e_{a}, e_{b}, F_{\mu \nu}^{c} e_{c}\right] F^{\mu \nu n} e_{n}>= \\
-\frac{1}{4 \kappa^{2}} \int d^{D} x \Lambda^{a b} F_{\mu \nu}^{c} F^{\mu \nu n}\left(<e_{c} f_{a b n k} e_{k}>+<f_{a b c k} e_{k} e_{n}>\right)=0 .
\end{gathered}
$$

this is a direct result of

$<e_{c} f_{a b n k} e_{k}>+<f_{a b c k} e_{k} e_{n}>=-\left(f_{a b n k} \delta_{c k}+f_{a b c k} \delta_{k n}\right)=-\left(f_{a b n c}+f_{a b c n}\right)=0$

due to the property $f_{a b n c}+f_{a b c n}=0$ which can be explicitly verified as follows

$$
\left[d_{a b n c}+2 \delta_{a n} \delta_{b c}-2 \delta_{b n} \delta_{a c}\right]+\left[d_{a b c n}+2 \delta_{a c} \delta_{b n}-2 \delta_{b c} \delta_{a n}\right]=0
$$

because $d_{a b n c}+d_{a b c n}=0 ; d_{n a b c}+d_{c a b n}=0$, due to the total antisymmetry of the associator structure constant $d_{n a b c}$ under the exchange of any pair of indices. A shortcut to prove the invariance $\delta S=0$ is simply $\delta\left(F_{\mu \nu}^{k}\right)^{2}=$ $2 \Lambda^{i j} f_{i j}{ }^{l k} F_{\mu \nu l} F_{k}^{\mu \nu}=0$ due to the antisymmetry $f_{i j}{ }^{l k}=-f_{i j}{ }^{k l}$ and symmetry $F_{\mu \nu l} F_{k}^{\mu \nu}=F_{\mu \nu k} F_{l}^{\mu \nu}$ under the exchange of indices $k \leftrightarrow l$. The variation of the components associated with the $e_{0}$ generator is trivially zero $\delta\left(F_{\mu \nu}^{0}\right)^{2}=0$ because $\left[e_{i}, e_{j}, e_{0}\right]=0$.

This work is not complete until we show the closure of the infinitesimal transformations (2.23). To achieve this one needs first to recast them as derivations

$$
\begin{aligned}
& \delta_{1} A_{\mu}=\delta_{1}\left(A_{\mu}^{k} e_{k}\right)=\Lambda_{1}^{a b}\left[e_{a}, e_{b}, A_{\mu}^{c} e_{c}\right]=\Lambda_{1}^{a b} D_{e_{a}, e_{b}} A_{\mu} \\
& \delta_{2} A_{\mu}=\delta_{2}\left(A_{\mu}^{k} e_{k}\right)=\Lambda_{2}^{c d}\left[e_{c}, e_{d}, A_{\mu}^{l} e_{l}\right]=\Lambda_{2}^{c d} D_{e_{c}, e_{d}} A_{\mu}
\end{aligned}
$$

by recurring to the fundamental identity (2.10) in order to evaluate the commutator of two derivations, and after relabeling indices, one arrives at

$$
\begin{gathered}
{\left[\delta_{1}, \delta_{2}\right] A_{\mu}=\Lambda_{2}^{c d} \Lambda_{1}^{a b}\left[D_{e_{c}, e_{d}}, D_{e_{a}, e_{b}}\right] A_{\mu}=} \\
\Lambda_{2}^{c d} \Lambda_{1}^{a b}\left(D_{\left[e_{c}, e_{d}, e_{a}\right], e_{b}}+D_{\left.e_{a}, e_{c}, e_{d}, e_{b}\right]}\right) A_{\mu}= \\
\Lambda_{2}^{c d} \Lambda_{1}^{a b}\left(\left[\left[e_{c}, e_{d}, e_{a}\right], e_{b}, A_{\mu}\right]+\left[e_{a},\left[e_{c}, e_{d}, e_{b}\right], A_{\mu}\right]\right)= \\
\Lambda_{2}^{c d} \Lambda_{1}^{a b} f_{c d a k}\left[e_{k}, e_{b}, A_{\mu}\right]+\Lambda_{2}^{c d} \Lambda_{1}^{a b} f_{c d b k}\left[e_{a}, e_{k}, A_{\mu}\right]= \\
-\left(\Lambda_{2}^{c d} \Lambda_{1}^{a k} f_{c d a}^{b}-\Lambda_{2}^{c d} \Lambda_{1}^{a b} f_{c d a}^{k}\right)\left[e_{k}, e_{b}, A_{\mu}\right]=\Lambda_{3}^{k b}\left[e_{k}, e_{b}, A_{\mu}\right]=\delta_{3} A_{\mu}
\end{gathered}
$$

Therefore the antisymmetric parameter resulting from the closure of two transformations is given by

$$
\Lambda_{3}^{k b}=-\left(\Lambda_{2}^{c d} \Lambda_{1}^{a k} f_{c d a}^{b}-\Lambda_{2}^{c d} \Lambda_{1}^{a b} f_{c d a}^{k}\right)
$$


Inserting the solutions given by eq-(2.29b) into (2.41) give

$$
\Lambda_{3}^{k b}=-\left(C_{2}^{c d} C_{1}^{a k} f_{c d a}^{b}-C_{2}^{c d} C_{1}^{a b} f_{c d a}^{k}\right) e^{\left(\lambda_{1}+\lambda_{2}\right) \phi}=C_{3}^{k b} e^{\lambda_{3} \phi}
$$

where $\lambda_{3}=\lambda_{1}+\lambda_{2}$ and the constant entries of the antisymmetric $\mathbf{C}_{3}$ matrix are given in terms of the constant entries of the antisymmetric matrices $\mathbf{C}_{1}, \mathbf{C}_{2}$ as follows

$$
C_{3}^{k b}=-\left(C_{2}^{c d} C_{1}^{a k} f_{c d a}^{b}-C_{2}^{c d} C_{1}^{a b} f_{c d a}^{k}\right)=-\left(\tilde{C}_{2 a}^{b} C_{1}^{a k}-\tilde{C}_{2 a}^{k} C_{1}^{a b}\right)
$$

Therefore, because the solution for $\Lambda_{3}^{k b}=C_{3}^{k b} e^{\lambda_{3} \phi}$ given by (2.42) has the same functional form as that required by eq- $(2.29 \mathrm{~b})$ in order to solve eq- $(2.25)$, there is closure of two gauge transformations as shown in the relations $(2.42,2.43)$ $\left(\lambda_{3}=\lambda_{1}+\lambda_{2}\right)$ among the parameters $\Lambda_{1}^{i j}, \Lambda_{2}^{i j}$ and $\Lambda_{3}^{i j}$. One may write such relation among the parameters symbolically as $\Lambda_{3}=\Lambda_{1} \wedge \Lambda_{2}$ such that $\left[\delta_{1}, \delta_{2}\right] A_{\mu}=\delta_{3} A_{\mu}$. follows

The finite ternary transformations can be obtained by "exponentiation" as

$$
F^{\prime}=F+\delta F+\frac{1}{2 !} \delta(\delta F)+\frac{1}{3 !}(\delta(\delta(\delta F)))+\ldots
$$

where $\delta\left(F_{\mu \nu}^{m} e_{m}\right)=\Lambda^{a b}\left[e_{a}, e_{b}, F_{\mu \nu}^{c} e_{c}\right] ; \delta(\delta F)=\Lambda^{m n}\left[e_{m}, e_{n}, \Lambda^{a b}\left[e_{a}, e_{b}, F_{\mu \nu}^{c} e_{c}\right]\right]$; ..... To show that the action is invariant under finite ternary local transformations requires to follow a few steps. Firstly, one defines

$$
<x y>\equiv \operatorname{Real}[\bar{x} y]=\frac{1}{2}(\bar{x} y+\bar{y} x) \Rightarrow<x y>=<y x>
$$

Despite nonassociativity, the very special conditions

$$
x(\bar{x} u)=(x \bar{x}) u ; \quad x(u \bar{x})=(x u) \bar{x} ; \quad x(x u)=(x x) u ; \quad x(u x)=(x u) x
$$

are obeyed for octonions resulting from the Moufang identities. Despite that $(x y) z \neq x(y z)$ one has that their real parts obey

$$
\operatorname{Real}[(x y) z]=\operatorname{Real}[x(y z)]
$$

Due to the nonassociativity of the algebra, in general one has that $(U F) U^{-1} \neq$ $U\left(F U^{-1}\right)$. However, if and only if $U^{-1}=\bar{U} \Rightarrow \bar{U} U=U \bar{U}=1$, as a result of the the very special conditions (2.46) one has that $F^{\prime}=(U F) U^{-1}=U\left(F U^{-1}\right)=$ $U F U^{-1}=U F \bar{U}$ is unambiguously defined. One can equate the result of the exponentiation procedure in eq-(2.44) to the expression

$$
F^{\prime}=U F U^{-1}=U F \bar{U}=e^{\Sigma^{k}\left(\Lambda^{a b}\right) e_{k}}\left(F^{c} t_{c}\right) e^{-\Sigma^{k}\left(\Lambda^{a b}\right) e_{k}} ; \quad k=1,2,3, \ldots, 7 .
$$

where $\Sigma^{k}\left(\Lambda^{a b}\right) e_{k}$ is a complicated function of $\Lambda^{a b}$. It yields the finite transformations which agree with the infinitesimal ternary ones when $\Lambda^{a b}$ are infinitesimals. 
For instance, to lowest order in $\Lambda^{a b}$, one has that $\Sigma^{k}$ satisfies $2 \Sigma^{k} c_{k c d}=\Lambda^{a b} f_{a b c d}$ and which follows by comparing the transformations in (2.44) to those in (2.48), to lowest order.

Dropping the spacetime indices for convenience in the expressions for $F^{\mu \nu}, F_{\mu \nu}$, and by repeated use of eqs- $(2.45,2.46,2.47)$, when $U^{-1}=\bar{U}$, the action density is also invariant under (unambiguously defined) transformations of the form $F^{\prime}=U F U^{-1}=U F \bar{U}$,

$$
\begin{aligned}
& <F^{\prime} F^{\prime}>=\operatorname{Re}\left[\bar{F}^{\prime} F^{\prime}\right]=\operatorname{Re}\left[\left(U \bar{F} U^{-1}\right)\left(U F U^{-1}\right)\right]=\operatorname{Re}\left[(U \bar{F})\left(U^{-1}\left(U F U^{-1}\right)\right)\right]= \\
& \operatorname{Re}\left[(U \bar{F})\left(U^{-1} U\right)\left(F U^{-1}\right)\right]=\operatorname{Re}\left[(U \bar{F})\left(F U^{-1}\right)\right]=\operatorname{Re}\left[\left(F U^{-1}\right)(U \bar{F})\right]= \\
& \operatorname{Re}\left[F\left(U^{-1}(U \bar{F})\right)\right]=\operatorname{Re}\left[F\left(U^{-1} U\right) \bar{F}\right]=\operatorname{Re}[F \bar{F}]=\operatorname{Re}[\bar{F} F]=<F F>.
\end{aligned}
$$

The real part of the coupling $g^{0}$ is inert under the transformations (2.23b) and it decouples from the definition of the field strength $F_{\mu \nu}$ because $e_{0}$ has a vanishing 3-bracket with other elements of the octonion algebra. The coupling $g^{0}=$ constant can be incorporated into the field strength in the same fashion as it occurs in ordinary Yang-Mills. One may rewrite the physical coupling $g^{0}$ as a prefactor in front of the 3-bracket as $F_{\mu \nu}=\partial_{\mu} A_{\nu}-\partial_{\nu} A_{\mu}+$ $g^{0}\left[A_{\mu}, A_{\nu}, \mathbf{g}\right]$, and reabsorb $g^{0}$ into the definition of the $A_{\mu}$ field as $F_{\mu \nu}=$ $\frac{1}{g^{0}}\left(\partial_{\mu}\left(g^{0} A_{\nu}\right)-\partial_{\nu}\left(g^{0} A_{\mu}\right)+\left[g^{0} A_{\mu}, g^{0} A_{\nu}, \mathbf{g}\right]\right)$. Thus $F_{\mu \nu} \rightarrow \frac{1}{g^{0}} F_{\mu \nu}$ and the action is rescaled as $S \rightarrow \frac{1}{\left(g^{0}\right)^{2}} S$ as it is customary in the Yang-Mills action.

To conclude this work, when $A_{\mu}^{i}=g^{i} \partial_{\mu} \phi$ we have an action ${ }^{1}$

$$
S=-\frac{1}{4 \kappa^{2}} \int d^{D} x\left(F_{\mu \nu}^{0} F_{0}^{\mu \nu}-F_{\mu \nu}^{i} F_{i}^{\mu \nu}\right), \quad i=1,2,3, \ldots ., 7
$$

the $\left(g^{0}\right)^{2}$ coupling squared can be reabsorbed into the definition of $\kappa^{2}$. The kinetic terms are explicitly given as

$$
\begin{gathered}
F_{\mu \nu}^{i} F_{i}^{\mu \nu}=\left[\partial_{[\mu} A_{\nu]}^{l}+A_{\mu}^{i} A_{\nu}^{j} g^{k} f_{i j k}^{l}\right]^{2}=\left[\left(\partial_{\mu} g^{i}\right)\left(\partial_{\nu} \phi\right)-\left(\partial_{\nu} g^{i}\right)\left(\partial_{\mu} \phi\right)\right]^{2}= \\
2\left(\left(\partial_{\mu} g^{i}\right)^{2}\left(\partial_{\nu} \phi\right)^{2}-\left(\partial_{\mu} g^{i}\right)\left(\partial^{\mu} \phi\right)\left(\partial_{\nu} g_{i}\right)\left(\partial^{\nu} \phi\right)\right) \neq 0 \\
F_{\mu \nu}^{0} F_{0}^{\mu \nu}=\left(\partial_{\mu} A_{\nu}^{0}-\partial_{\nu} A_{\mu}^{0}\right)\left(\partial^{\mu} A_{0}^{\nu}-\partial^{\nu} A_{0}^{\mu}\right)
\end{gathered}
$$

and which are invariant under the transformations $(2.23 \mathrm{a}, 2.23 \mathrm{~b})$ due to eqs$(2.27)$ and $\delta \phi=0(2.34)$. The solutions to eqs- $(2.25,2.27)$ are provided by eqs- $(2.30,2.31 \mathrm{~b})$. It would have been desirable to avoid the conditions $(2.25$, 2.27 ) which force the gauge parameters $\Lambda^{i j}(x)$ to be field-dependent in the sense that they are given by eq-(2.31b) and which has a Wilson-loop-like expression. There is closure of two gauge transformations as indicated by eqs-(2.40-2.43).

The nontrivial role of the $7+1$ scalars in the action (2.51) given by the coupling functions $g^{i}(x)$ and $\phi(x)$ warrants to be studied further. We should

\footnotetext{
${ }^{1}$ One can choose $\int\left\langle F_{\mu \nu}^{*} F^{\mu \nu}>\right.$ for the action so that the $F_{\mu \nu}^{i} F_{i}^{\mu \nu}$ terms also appear with a positive sign inside the integrand.
} 
emphasize that the kinetic terms $F_{\mu \nu}^{i} F_{i}^{\mu \nu}$ for the latter scalars do not appear in the action in the usual canonical form $\left(D_{\mu} \Theta^{i}\right)^{2}$. One reason being that $D_{\mu} \Theta^{l}=\partial_{\mu} \Theta^{l}+A_{\mu}^{i} \Theta^{j} g^{k} f_{i j k}^{l}$ does not transform homogeneously under gauge transformations unless the gauge parameters are trivially constant. However, when the scalars $g^{i}(x), \phi(x)$ appear in the action in the form displayed by eqs$(2.50,2.51)$, the $F_{\mu \nu}^{i}$ terms transform homogeneously $\delta F_{\mu \nu}^{l}=\Lambda^{i j} F_{\mu \nu}^{k} f_{i j k}^{l}$ as they should, whereas $\delta F_{\mu \nu}^{0}=0$.

The terms (2.52) have the same functional form as the Maxwell action but the transformations laws for $A_{\mu}^{0}\left(\delta A_{\mu}^{0}=0\right)$ differ from the $U(1)$ gauge field ( $\delta \mathcal{A}_{\mu}=\partial_{\mu} \Lambda$ ). The inclusion of potential terms for the scalar fields and ChernSimons actions will be the subject of future investigation. These octonionc ternary gauge field theories deserve further investigation. In particular, to study their relation to Yang-Mills theories based on the $G_{2}$ group which is the automorphism group of the Octonions and their relevance to Noncommutative and Nonassociative Geometry.

\section{Acknowledgments}

We thank M. Bowers for her assistance.

\section{References}

[1] P. Jordan, J von Neumann and E. Wigner, Ann. Math 35 ( 1934 ) 2964.

[2] K. MacCrimmon, "A Taste of Jordan Algebras" ( Springer Verlag, New York 2003).

[3] H. Freudenthal, Nederl. Akad. Wetensch. Proc. Ser 57 A (1954) 218.

[4] J. Tits, Nederl. Akad. Wetensch. Proc. Ser 65 A (1962 ) 530.

[5] T. Springer, Nederl. Akad. Wetensch. Proc. Ser 65 A (1962 ) 259.

[6] J. Adams, " Lectures on Exceptional Lie Groups" Chicago Lectures in Mathematics, (Univ. of Chicago Press, Chicago 1996).

[7] R. Schafer, "An introduction to Nonassociative Algebras" (Academic Press 1966).

[8] C. H Tze and F. Gursey, "On the role of Divison, Jordan and Related Algebras in Particle Physics" World Scientific 1996.

[9] S. Okubo, Introduction to Octonion and other Nonassociative Algebras in Physics" (Cambridge Univ. Press, 2005).

[10] Y. Nambu, Phys. D 7 (1973) 2405.

[11] T. Springer and F. Veldkamp, " Octonions, Jordan Algebras and Exceptional Groups" (Springer Verlag 2000) 
[12] G. Dixon," Division Algebras, Octonions, Quaternions, Complex Numbers, and the Algebraic Design of Physics" ( Kluwer, Dordrecht, 1994).

[13] G. Dixon, J. Math. Phys 45 , no 10 (2004) 3678.

[14] J. Baez," The Octonions" Bull. Amer. Math. Soc. 39 (2002), 145-205, [arXiv : math.RA/0105155].

[15] R. Kerner, " Ternary Algebraic Structures and their applications in Physics" Proceedings of the Conference ICGTMP "Group-23", Dubna, Russia, July 30 - August 6, 2000[arXiv : math-ph/0011023] .

[16] R. Kerner, Communications in Math. Phys. 91 (1983) 213;

[17] R. Kerner, Classical and Quantum Gravity 14 (1A) (1997) 203.

[18] M. Dubois-Violette, R. Kerner and J. Madore, Jour Math Phys 31 (1990) 316; ibid (1990) 323.

[19] M. Dubois-Violette and M.Henneaux, Communications in Math. Phys. 226 (2002) 393;

[20] Y. Kawamura, Prof. Theor. Phys. 110, 579 (2003).

[21] P.-M. Ho, R.-C. Hou and Y. Matsuo, JHEP 0806 (2008) 020. P.-M. Ho, "Nambu Bracket for M Theory" arXiv:0912.0055 [hep-th].

[22] J. P. Gauntlett and J. B. Gutowski, ?Constraining Maximally Supersymmetric Membrane Actions,? arXiv:0804.3078 [hep-th].

[23] G. Papadopoulos, JHEP 0805 (2008) 054.

[24] M. Yamazaki, " Octonions, $G_{2}$ and generalized Lie 3-algebras " Phys. Letts B $670(2008) 215$.

[25] G. Dixon," Division algebras, Spinors, Idempotents, the Algebraic structure of Reality" arXiv : 1012.1304.

"Octonions " $E_{8}$ Lattice to $\Lambda_{16} "$ arXiv : hep-th/9501007.

[26] L. Boya, "Octonions and M-theory" (Talk Presented at the the 24th International Colloquium on Group-Theoretical Methods in Physics. Paris, July 15-22, 2002) arXiv:hep-th/0301037.

[27] J. Bagger and N. Lambert, Phys. Rev. D 75 : 045020 (2007);

[28] J. Bagger and N. Lambert, Phys. Rev D 77 : 065008 (2008); JHEP 02 : 105 (2008).

[29] A. Gustavsson, JHEP 04 : 083 (2008); Nuc. Phys B 811 (2009) 66.

[30] D. Jafferis, O. Aharony, O. Bergman and J. Maldacena, JHEP 10 : 091 (2008). 
[31] J. Bagger and N. Lambert, "Three-Algebras and N=6 Chern-Simons Gauge Theories," arXiv:0807.0163 [hep-th].

[32] P. de Medeiros, J. Figueroa-O?Farrill, E. Mendez-Escobar and P. Ritter, "On the Lie-algebraic origin of metric 3-algebras," arXiv:0809.1086 [hepth].

[33] T. Ootsuka, E. Tanaka and E. Loginov, "Nonassociative Gauge Theory" [arXiv : hep-th/0512349] .

[34] E. Loginov, Nucl. Phys B 606 (2001) 636.

[35] R. Foot and G. Joshi, Phys. Rev D 36 (1987) 1169; Int. J. of Theor. Phys 28, no. 3 (1989) 263.

[36] V. Dzhunushaliev, "Nonassociative generalization of Supersymmetry" arXiv.org : 1302.0346.

J. Gen. Lie. Theory and Applications, vol 2, no. 4 (2008) 269.

J. Gen. Lie. Theory and Applications, vol 3, no. 1 (2009) 33.

[37] L. Borsten, D. Dahanayake, M. J. Duff, H. Ebrahim and W. Rubens, " Black Holes, Qubits and Octonions" arXiv : 0809.4685.

[38] C. Castro, Int. J. Mod. Phys A 26, no. 18 (2011) 2997-3012 